\title{
High-accuracy function synthesizer circuit with applications in signal processing
}

\author{
Cosmin Popa
}

\begin{abstract}
An original low-voltage current-mode high-accuracy function synthesizer circuit will be presented, allowing to implement a multitude of continuous mathematical functions. The dynamic range is strongly extended as a result of the superior-order approximation of the implemented functions. The current-mode operation and the independence of the circuit performances on technological parameters are responsible for an additional improvement of structure accuracy. The advantages of reduced design costs per function represent an immediate consequence of the multiple functions realized by the proposed structure. The approximation error of the original function synthesizer circuit is $0.3 \%$ for an extended range of the input signal. The function synthesizer is designed for implementing in $0.18 \mu \mathrm{m}$ CMOS technology and it is supplied at $1 \mathrm{~V}$. An original application of the proposed function synthesizer circuit is represented by a new fourth-order approximation exponential function generator, having a dynamic range of approximately $33 \mathrm{~dB}$, for an error smaller than $1 \mathrm{~dB}$.
\end{abstract}

Keywords: Signal synthesizing, Approximation error, Exponential function generator, Current-mode operation, Continuous mathematical function, CMOS analog designs

\section{Introduction}

Analog signal processing represents an important area of analog integrated circuits analysis and design, with a multitude of applications in many domains. Multiplier and exponential circuits are useful in telecommunication circuits [1-4], medical equipments [5,6], hearing devices $[7,8]$, or disk drives $[9,10]$. Squaring circuits represent the core for implementing any continuous function, using the limited Taylor series expansion. The Euclidean distance function is very important in instrumentation circuits [11,12], communication [1,2], neural networks [13,14], display systems $[15,16]$, or classification algorithms [17], being also useful for vector quantization or nearest neighbor classification $[18,19]$.

The main problem in designing analog signal processing structures is how to implement with minimal effort a large number of nonlinear mathematical functions [3]. The requirements for an analog signal processing structure are mainly related to the circuit accuracy, to the possibility of achieving a multitude of circuit functions with reasonable design costs and to the controllability of the implemented

Correspondence: cosmin_popa@yahoo.com

Faculty of Electronics, Telecommunications and Information Technology, University Politehnica of Bucharest, Bucharest, Romania function. In this context, analog processing performed in focal-plane Vision Systems-on-Chip [20] can represent an interesting choice. Important functions from the perspective of their applications are multiplying/dividing [21-30], exponential [31-34], squaring/square-rooting [30,35-41], or Euclidean distance [42,43] functions.

In bipolar technology, the multiplying/dividing function can easily be obtained from the logarithmical characteristic of the bipolar transistor. Important errors still remain because of the nonzero values of the base currents and of the temperature dependence of the bipolar transistor parameters (the thermal voltage is linearly increasing with temperature and the saturation current has an exponential dependence on temperature). In order to achieve a lowpower operation of complementary metal oxide semiconductor (CMOS) designs, the subthreshold biasing of metal oxide semiconductor (MOS) transistors is an interesting choice. Based on the logarithmical law of a MOS transistor in weak inversion, the implementation of a CMOS Multiplier/Divider circuit becomes very simple (even with respect to the bipolar version). In consequence, the result will be smaller silicon area consumption, the circuit being also compatible with low-power very large-scale integration (VLSI) designs. For obtaining an important increasing 
of the circuit frequency response, the multiplying/dividing function can be achieved by employing the square law model of MOS transistors biased in saturation.

The exponential function is available in bipolar technology using the exponential characteristic of the bipolar transistor. In CMOS technology, the exponential law can directly be implemented exploiting the weak inversion operation of the MOS transistor. In order to obtain the exponential function using the squaring characteristic of the MOS transistor in saturation (for improving the circuit frequency response), the classical method is to approximate the exponential function with its $n$ th-order expansion (the polynomial series). The approximation error will be proportional with the number of terms neglected in the expansion.

There are many possibilities of implementing squaring and square-rooting functions using the quadratic characteristic of the MOS transistor biased in saturation. The main goals of this class of circuits are the silicon-occupied area, the independence of the output current on the technological parameters and on temperature and the small sensitivity to the multitude of second-order effects.

The classical approach in analog signal processing circuits is to implement, for each circuit function, a class of computational structures. The proposed method for obtaining a multitude of continuous mathematical functions is to design a complex computational structure, named function synthesizer circuit that is able to generate these functions approximating them using an original superior-order approximation function. The accuracy of computation is correlated with the order of approximation. In conclusion, a tradeoff between the complexity of the structure and its intrinsic accuracy must be made. The advantages of the proposed method are mainly related to the possibility of strongly reduce the power consumption and circuit complexity per implemented function.

It exists in literature a relatively small number of function synthesizer circuits implemented in CMOS technology $[4,33,44-46]$, these applications being dedicated to the realization of a limited number of mathematical functions. In [44], the approximation of the implemented function can be obtained by adding the weighted output currents of a number of basic building blocks, built around a basic current squarer, and a constant current, the circuit presenting the disadvantage of a relatively large complexity. The circuit proposed in [45] is based on approximating the required function using the first three terms of its Taylor series expansion. The approximations can be implemented by adding the output currents of a weighted current square, a weighted current amplifier (or attenuator), and a constant current. The errors are mainly caused by the small value (two) of the approximation order and, in consequence, they are relatively large. Additionally, from the same reason, the range of the input signal is strongly restricted. A structure for synthesis of analog exponential functions, based on approximating the exponential function using rational functions, is proposed in [46]. The circuit presents the important limitations of realizing only the exponential function and of an implementation in bipolar technology.

The article is multidisciplinary, starting from a rigorous mathematical analysis and continuing with original implementations of the proposed electronic computational circuits. A new implementation of a current-mode function synthesizer circuit with an extended capability of generating continuous mathematical functions will be presented. The proposed structure is based on a fourthorder original approximation function, having the important advantages of an improved accuracy and of reconfiguration capability.

\section{Main text}

\section{Fundamental methodology}

In order to implement an improved accuracy analog function synthesizer circuit, the proposed method is to use a general continuous approximation function, $g(x)$, having a Taylor series that can be made to fourth-order match a multitude of continuous mathematical functions, $f(x)$.

$$
g(x)=\frac{1+a_{O} x}{1+a_{1} x}+\frac{a_{2} x}{1+x}+a_{3} x+a_{4}
$$

$a_{O} \ldots a_{4}$ represent constant coefficients, having values imposed by the necessity that the Taylor series of $g(x)$ function to be identical (in a fourth-order approximation) with the Taylor series of $f(x)$ function, that can be expressed by the following polynomial relation:

$$
f(x)=m+n x+p x^{2}+q x^{3}+r x^{4}+s x^{5}+t x^{6}+\ldots
$$

$m \div t$ represents constant coefficients of the expansion, depending on the expression of $f(x)$ function that must be implemented.

The motivation for choosing this particular $g(x)$ approximation function is correlated with the possibilities of its facile implementation in CMOS technology (the multiplying/dividing function has a relatively simple realization). In comparison with this original approximation function, the Pade approximation [24] (having also good accuracy) presents a much more complicated implementation in CMOS technology, requiring squaring and superior-order computational circuits that strongly increase the overall complexity of the function synthesizer. The requirements for an extremely accuracy of the function synthesizer circuit impose a relatively high order of approximation for $g$ $(x)$ function. The increasing of the order of approximation strongly increases the complexity of the designed circuit, 
being necessary to realize a tradeoff between the circuit complexity and its overall accuracy. From this point of view, an optimal choice that allows to obtain a very good accuracy and a relative large dynamic range of the function synthesizer using a reasonable circuit complexity is based on a fourth-order approximation.

The fourth-order identity between the Taylor series of $f(x)$ and $g(x)$ functions is equivalent with the identity between the first five terms from the Taylor series of the previous functions, being evident the reason of choosing five coefficients $a_{O} \ldots a_{4}$ for defining $g(x)$ function. It results

$$
\begin{aligned}
& a_{O}=\frac{(p+q)^{3}}{(r+q)(r+p+2 q)}-\frac{r+q}{p+q} \\
& a_{1}=-\frac{r+q}{p+q} \\
& a_{2}=\frac{(p+q)^{2}}{r+p+2 q}-p \\
& a_{3}=n+p-\frac{(p+q)^{2}}{r+q} \\
& a_{4}=m-1
\end{aligned}
$$

The approximation error will have the following general expression:

$$
\epsilon_{f(x)}^{g(x)}(x) \cong\left[\frac{q^{2}-2 r p-q r}{p+q}-s\right] \frac{x^{5}}{f(x)}
$$

In order to further reduce the approximation error of the synthesizer circuit, it is possible to increase the order of approximation. For example, a possible fifth-order approximation function that requires a reasonable complexity of CMOS implementation can be generally expressed as follows:

$$
g^{\prime}(x)=\frac{b_{O} x}{1+b_{1} x}+\frac{b_{2} x}{1+x}+b_{3} x^{2}+b_{4} x+b_{5}
$$

$b_{O} \ldots b_{4}$ represent constant coefficients, having values imposed by the necessity that the Taylor series of $g^{\prime}(x)$ function to be identical (in a fifth-order approximation) with the Taylor series (2) of $f(x)$ function. The additional complexity of the circuit that implements $g^{\prime}(x)$ function comparing with the computation structure required by $g^{\prime}(x)$ is represented by a current-mode squaring circuit (for obtaining the $b_{3} x^{2}$ term).
Current-mode implementation of the function synthesizer circuit

Block diagram of the function synthesizer with fourth-order approximation

The block diagram of the function synthesizer, based on the original proposed approximation function (1), is presented in Figure 1. The "Multiplier/Divider" circuits are current-mode structures $[30,47,48]$, having the new implementation and the description of their operation presented in the following paragraph. The expressions of $I_{\text {OUTa }}$ and $I_{\text {OUTb }}$ currents are

$$
I_{\text {OUTa }}=\frac{I_{O} I_{1 a}}{I_{2 a}}
$$

and

$$
I_{\text {OUTb }}=\frac{I_{O} I_{1 b}}{I_{2 b}}
$$

The output current of the circuit having the block diagram presented in Figure 1 will have the following expression:

$$
I_{\text {OUT }}=I_{\text {OUTa }}+I_{\text {OUTb }}+a_{3} I_{I N}+a_{4} I_{O}
$$

Using the notation $x=I_{I N} / I_{O}$ and relation (1), it results that $I_{O U T}$ current represents the fourth-order approximation of $f(x)$ function:

$$
\begin{aligned}
I_{\text {OUT }} & =I_{O}\left(\frac{1+a_{O} x}{1+a_{1} x}+\frac{a_{2} x}{1+x}+a_{3} x+a_{4}\right) \\
& =I_{O} g_{2}(x) \cong I_{O} f(x)
\end{aligned}
$$

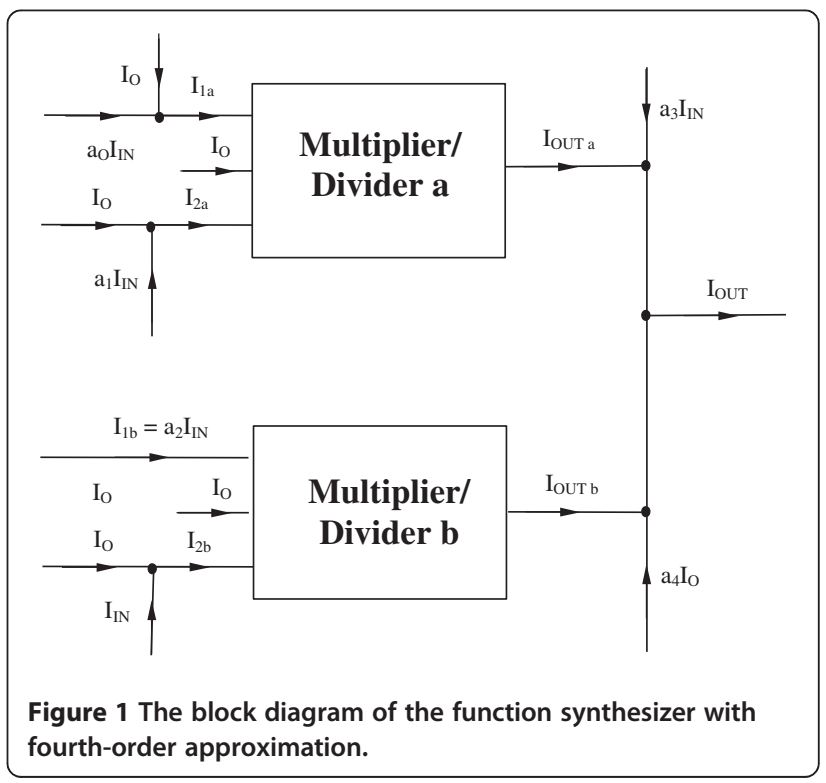




\section{The implementation of the " $\mathrm{X}^{2 \text { " block }}$}

The functional core for designing the "Multiplier/Divider" circuit is represented by a current-mode squaring circuit, having the original implementation presented in Figure 2. The aspect ratios of MOS transistors are $1 \mu$ / $0.54 \mu$ for M1-M4 transistors and $0.8 \mu / 0.54 \mu$ for M5M7 transistors.

Noting with $V_{G S}(I)$ the absolute value of the gate-source voltage of a MOS transistor biased at a drain current equal with $I$ the equation of the translinear loop can be expressed as follows:

$$
2 V_{G S}\left(I_{O}\right)=V_{G S}\left(I_{D}\right)+V_{G S}\left(I_{D}+I_{I N}\right)
$$

resulting

$$
2 \sqrt{I_{O}}=\sqrt{I_{D}}+\sqrt{I_{D}+I_{I N}}
$$

Reducing the radicals by squaring the terms and simplifying, it results

$$
4 I_{O}-2 I_{D}-I_{I N}=2 \sqrt{I_{D}\left(I_{D}+I_{I N}\right)}
$$

equivalent with:

$$
16 I_{O}^{2}+I_{I N}^{2}-16 I_{D} I_{O}-8 I_{O} I_{I N}=0
$$

So

$$
I_{D}=I_{O}-\frac{I_{I N}}{2}+\frac{I_{I N}^{2}}{16 I_{O}}
$$

The expression of the output current will be

$$
I_{O U T}=I_{D}+\frac{I_{I N}}{2}-I_{O}=\frac{I_{I N}^{2}}{16 I_{O}}
$$

The previous relations can be used only for strong saturated devices and for not too small devices. In order to

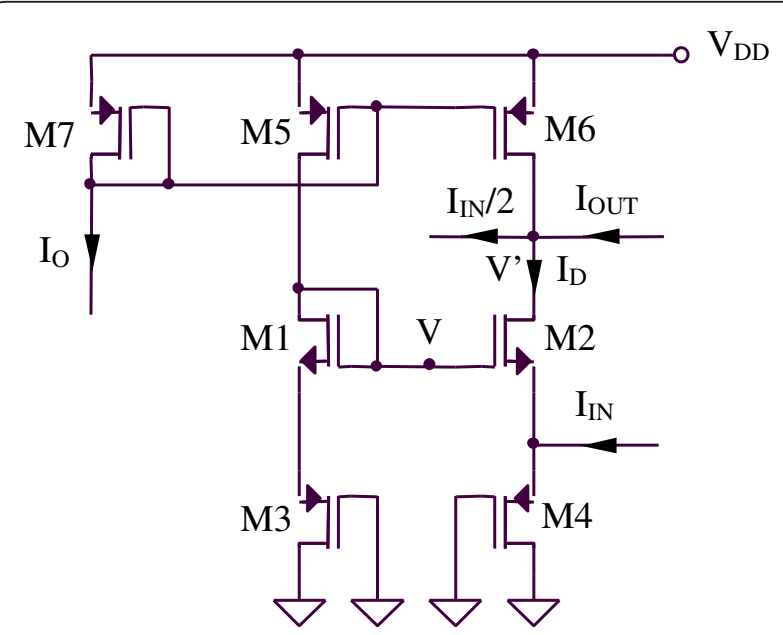

Figure 2 The implementation of the " $X$ " block. increase the accuracy of the computations, quasi-identical source-drain voltages for M1-M2 and M3-M4 transistors, respectively, must be imposed. The source-drain voltage of M3 transistor can be expressed as follows

$$
V_{S D 3}=V-V_{G S 1} \cong V-V_{G S 2}=V_{S D 4}
$$

because, considering $I_{I N}<<I_{O}$, M1 and M2 transistors are identical and biased at drain currents that differ only with a small amount. Additionally, for biasing M1 and M2 transistors at approximately equal drainsource voltages, $V$ potential must be imposed, from the $I_{O U T}$ external terminal, to be equal with $V$ potential. This particular biasing of M1 and M2 transistors will impose quasi-identical source-drain voltages also for M5 and M6 transistors, this fact increasing the accuracy of M5-M6 current mirror.

The M3-M7 transistors are not affected by the substrate effect, as their bulks are connected to their sources terminals. The M1 and M2 transistors have the bulk connected to the ground, so their non-zero bulksource voltage will be responsible for small errors introduced by the substrate effect. As a result, the expression of $I_{O U T}$ current will slightly be affected by undesired dependencies on technological parameters. The impact of these errors on the overall accuracy of the proposed function synthesizer circuit is small and they can be compensated using specific design techniques. The biasing current of the squaring circuit from Figure 2 is approximately $200 \mu A$.

\section{The implementation of the "multiplier/divider" block}

The original proposed "Multiplier/Divider" circuit is presented in Figure 3a, being designed using two currentmode squaring circuits, similar with the structures presented in Figure 2 (M1-M4 and M3-M6, respectively). The full CMOS implementation of the "Multiplier/Divider" circuit is shown in Figure 3b. The complexity of the "Multiplier/Divider" circuit is minimized by re-using M3-M4 transistors for both squaring circuits. The aspect ratios of MOS transistors are $1 \mu / 0.54 \mu$ for M1-M6 transistors and $0.8 \mu / 0.54 \mu$ for $\mathrm{M} 7-\mathrm{M} 8$ transistors. The output current has the following expression:

$$
I_{O U T}=I_{D 1}-I_{D 2}+2 I_{O}
$$

where two translinear loops similar with the loop ana-

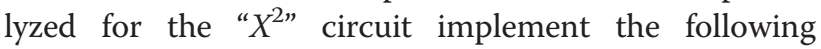
expressions of $I_{D 1}$ and $I_{D 2}$ currents (derived from (19)):

$$
I_{D 1}=I_{2}-\left(I_{1}+I_{O}\right)+\frac{\left(I_{1}+I_{O}\right)^{2}}{4 I_{2}}
$$




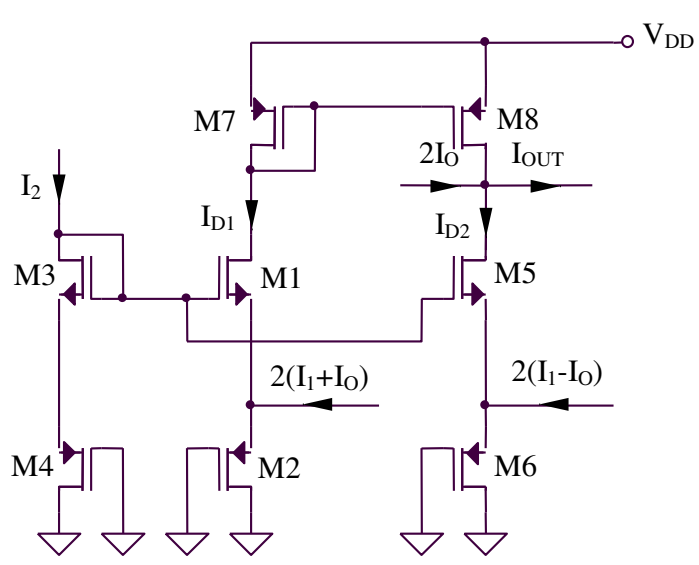

(a)

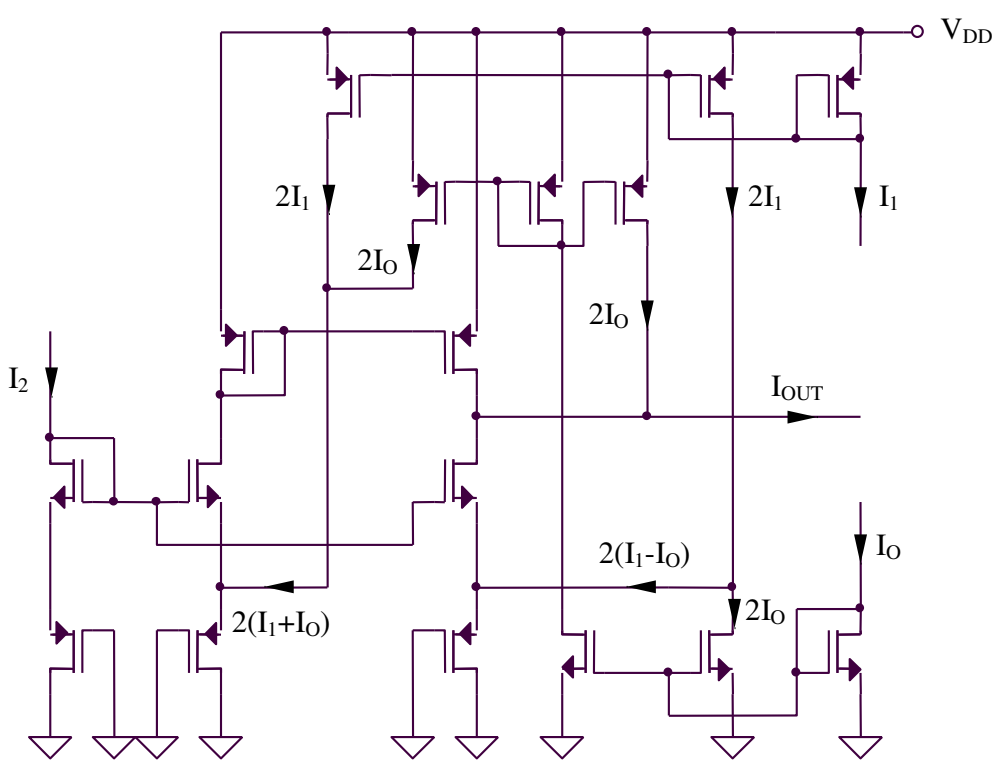

(b)

Figure 3 (a) The core of the Multiplier/Divider circuit. (b) The implementation of the Multiplier/Divider circuit.

$$
I_{D 2}=I_{2}-\left(I_{1}-I_{O}\right)+\frac{\left(I_{1}-I_{O}\right)^{2}}{4 I_{2}}
$$

resulting an output current of the circuit having the following expression:

$$
I_{\text {OUT }}=I_{O} \frac{I_{1}}{I_{2}}
$$

The original proposed Multiplier/Divider circuit presents two important advantages. First, the current-mode operation increases the frequency response of the computational structure. Second, the independence of the output current expressed by (24) on technological parameters removes, in a first-order analysis, the temperature- and technological-caused errors, the overall accuracy of the proposed circuit being increased in this way. Additionally, the resulted concrete implementation of the function synthesizer circuit based on the block diagram proposed in Figure 4 has a smaller complexity comparing with previously reported similar computational structures.

Using MOS transistors implemented in $0.18 \mu \mathrm{m}$ CMOS technology, the maximal frequency of operation of the proposed function synthesizer circuit is approximately hundreds $\mathrm{kHz}-\mathrm{MHz}$, depending on the particular model of MOS active devices. The biasing current of the "Multiplier/Divider" circuit is approximately $300 \mu \mathrm{A}$, while the maximal biasing current of the function synthesizer circuit is smaller than $800 \mu \mathrm{A}$.

\section{Error mechanisms for function synthesizer circuit}

Real circuits are affected by a multitude of errors [3,4] that slightly affects their overall accuracy. The most 


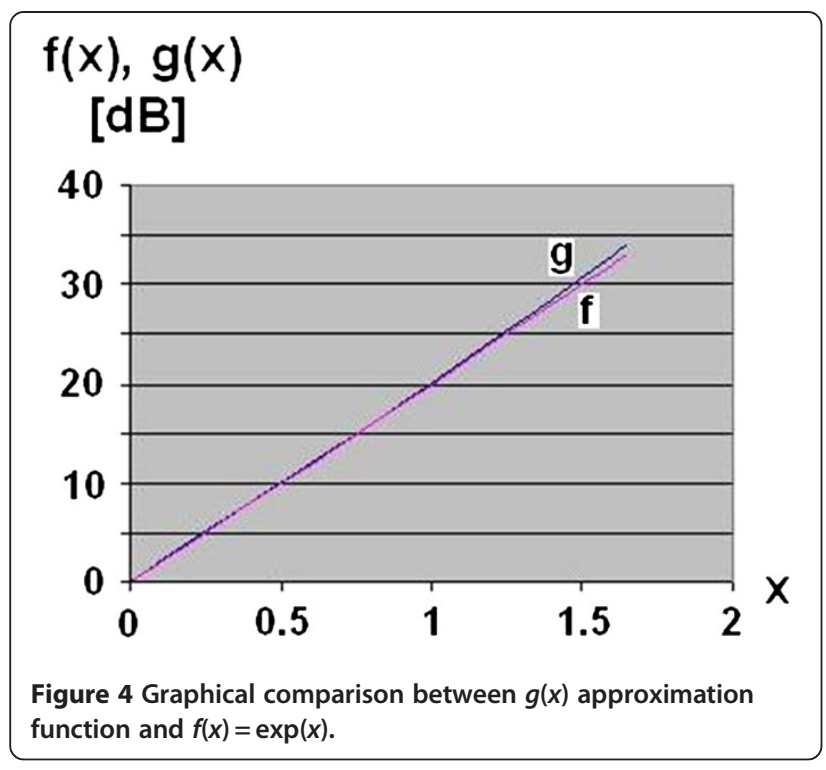

important errors that must be taken into account for evaluating the function synthesizer accuracy are presented in the following paragraphs.

\section{The deviation of the MOS transistor characteristic from the square-law, bulk effect, leakage}

The saturated MOS transistor squaring characteristic is affected by the second-order effects: mobility degradation (25), channel-length modulation (26), and bulk effect (27):

$$
\begin{aligned}
& K=\frac{K_{O}}{\left[1+\theta_{G}\left(V_{G S}-V_{T}\right)\right]\left(1+\theta_{D} V_{D S}\right)} \\
& I_{D}=\frac{K}{2}\left(V_{G S}-V_{T}\right)^{2}\left(1+\lambda V_{D S}\right) \\
& V_{T}=V_{T O}+\gamma\left(\sqrt{\Phi-V_{B S}}-\sqrt{\Phi}\right)
\end{aligned}
$$

Table 1 Comparison between $g(x)$ approximation function and $f(x)=\exp (x)$

\begin{tabular}{llll}
\hline $\boldsymbol{x}$ & $\boldsymbol{g}(\boldsymbol{x})(\mathbf{d B})$ & $\boldsymbol{f}(\boldsymbol{x})(\mathbf{d B})$ & $\boldsymbol{\epsilon}(\mathbf{d B})$ \\
\hline 0 & 0 & 0 & 0 \\
0.2 & 4.075 & 4 & 0.075 \\
0.4 & 8.029 & 8 & 0.029 \\
0.6 & 12.011 & 12 & 0.011 \\
0.8 & 16.028 & 16 & 0.028 \\
1.0 & 20.095 & 20 & 0.095 \\
1.2 & 24.239 & 24 & 0.239 \\
1.4 & 28.495 & 28 & 0.495 \\
1.6 & 32.914 & 32 & 0.914 \\
1.65 & 34.001 & 33 & 1.001 \\
\hline
\end{tabular}

The errors introduced by the bulk effect $[3,4]$ can be reduced by proper designs that avoid the dependence of the circuit parameters on the threshold voltage. The design of circuits for obtaining a zero bulk-source voltage cancels out the errors introduced by the bulk effect. Additional errors produced by the second-order effects are given by the dependence of $K$ transconductance parameter on $V_{G S}$ voltage. Taking into account only the $K\left(V_{G S}\right)$ dependence, a small changing of $K\left(V_{G S}\right)$ voltage comparing with the analysis based on the first-order model of MOS transistors can be determined:

$$
V_{G S}=V_{T}+\sqrt{\frac{2 I_{D}}{K}}+\frac{\theta_{G}}{K} I_{D}
$$

For latest CMOS VLSI nanometer designs, the leakage $[3,4]$ becomes more important, the leakage current depending on the properties of the layout and also on the device structure. The determination of the total leakage cannot be made by adding individual leakage currents, because of a correlation that exists between leakage currents.

The subthreshold leakage is the current produced by minority electrons flowing through $p$ substrate from source to drain, being modeled by an exponential function:

$$
I_{S L}=I_{D O} \frac{W}{L} \exp \left(\frac{V_{G S}-V_{T}}{n V_{T H}}\right)\left[1-\exp \left(-\frac{V_{D S}}{V_{T H}}\right)\right]
$$

$W / L$ is the aspect ratio of the MOS transistor, $V_{T H}$ is the thermal voltage, $n$ is a parameter, and $I_{D O}$ current is an additional parameter. The subthreshold-off current (obtained for $V_{G S}=0$ and $V_{D S}>>V_{T}$ ) can be expressed as follows:

$$
I_{S L o f f}=I_{D O} \frac{W}{L} \exp \left(\frac{-V_{T}}{n V_{T H}}\right)
$$

The gate leakage current can be carried by tunneling electrons or holes, the carriers leaking to source, drain, and channel. For junction leakage, low currents are carried by minority carriers drifting across the junction, by the electron-hole generation in junction or by the impact ionization at high reverse bias, the electrons being able to pass the barrier by tunneling through it-Band-To-Band Tunneling current $[3,4]$.

\section{Mismatches in current mirrors}

Current mirrors implemented using real circuits present some errors caused by the mismatches between the 


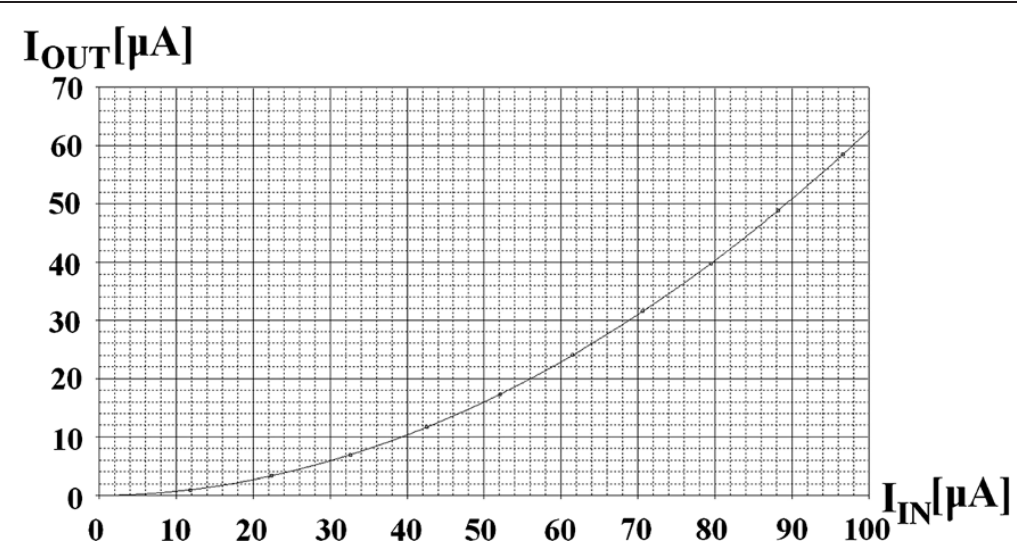

Figure 5 SPICE simulation $I_{\text {OUT }}\left(I_{\text {IN }}\right)$ for the squaring circuit.

composing MOS transistors and also by the channellength modulation:

$$
\frac{I_{O}}{I_{R E F}}=\frac{(W / L)_{O}}{(W / L)_{R E F}} \frac{1+\lambda V_{D S O}}{1+\lambda V_{D S R E F}}
$$

The method for reducing the errors introduced by the channel-length modulation is to impose to the current mirror an output voltage that set $V_{D S O} \cong V_{D S R E F}$.

\section{Different threshold voltages and $K_{n} / K_{p}$ constants for NMOS/ PMOS devices}

Real circuits present differences between the threshold voltages and $K_{n} / K_{p}$ constants for NMOS and PMOS transistors. In order to avoid additional inaccuracies introduced by the practical situation, translinear loops that represent the functional basis of many computational circuits must contain only NMOS or PMOS active devices or must be implemented using quasi-symmetrical structures from the point of view of this complementarily (the same number of NMOS and PMOS transistors).

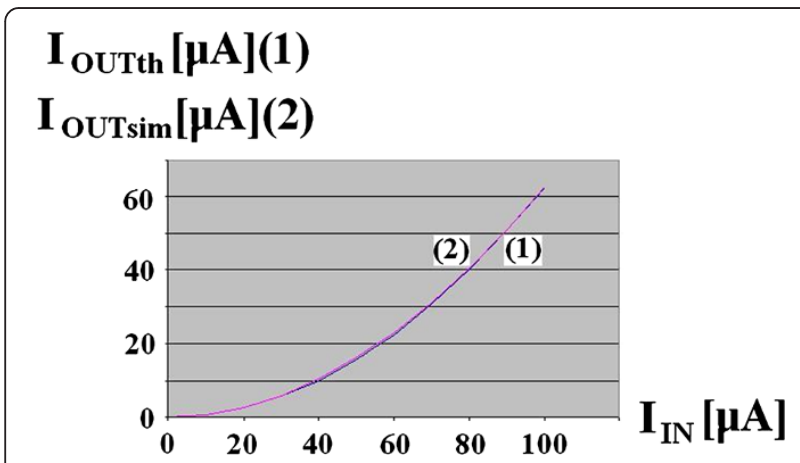

Figure 6 Comparison between the simulated and the theoretical estimated results for the current squarer.

\section{Variations over the process, temperature, and supply voltage}

Most of MOS transistors parameters are affected by the process in which the computational circuits are implemented. On the other side, technological parameters present important temperature dependencies. From this perspective, in order to avoid important errors introduced by process and temperature, the practical realizations of analog computational circuits must be done in such a way that minimizes the number of technological parameters in the expression of the output signal. Additionally, the errors caused by the supply voltage variations can be minimized using self-biased cascode configurations. In this context, a tradeoff between power supply rejection ratio and minimal supply voltage has to be considered.

\section{Applications}

An original exponential function generator circuit can be designed using the fourth-order approximation function (1), replacing in general relations (3)-(7) the particular values of constant coefficients of expansion (2): $m=1, n=1, p=1 / 2, q=1 / 6$ and $r=1 / 24$. The $g(x)$ function that fourth-order approximates the exponential function can be expressed as follows:

$$
g(x)=\frac{16+21 x}{16-5 x}+\frac{1}{126} \frac{1}{1+x}-\frac{7 x}{11}
$$

Using (8), the approximation error of the exponential function generator will have the following general expression:

$$
\epsilon_{\exp (x)}^{g(x)}(x) \cong \frac{x^{5}}{120 \exp (x)}
$$

A comparison between $g(x)$ approximation function and $f(x)=\exp (x)$ is shown in Table 1 . 


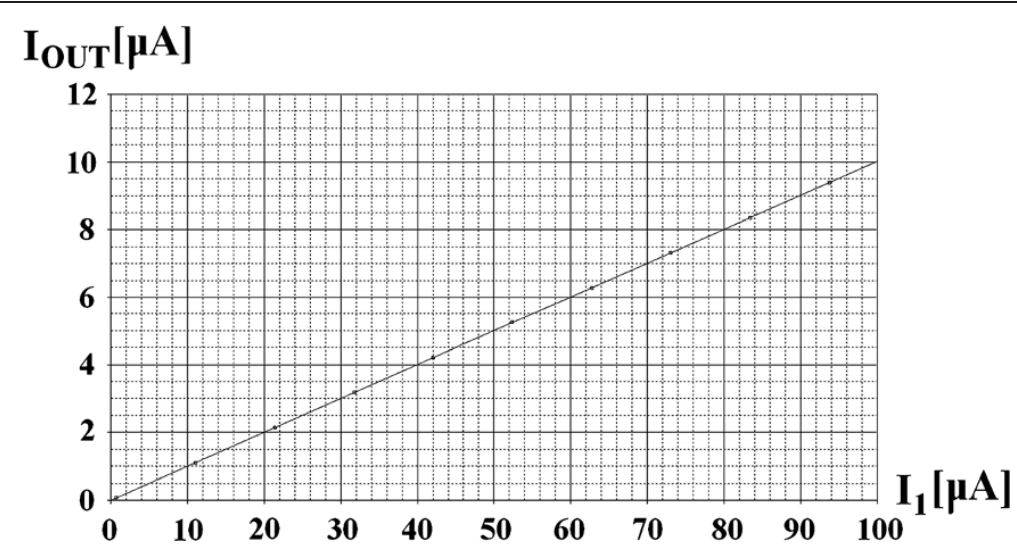

Figure 7 SPICE simulation $I_{\mathrm{OUT}}\left(I_{1}\right)$ for the Multiplier/Divider circuit.

A graphical comparison between $g(x)$ approximation function and $f(x)=\exp (x)$ is presented in Figure 4 .

As a result of using a fourth-order approximation function, the dynamic range of the proposed exponential function generator is approximately $33 \mathrm{~dB}$, for an error smaller than $1 \mathrm{~dB}$.

\section{Simulations}

The proposed function synthesizer circuit is designed for implementing in $0.18 \mu \mathrm{m}$ generic CMOS technology, the simulations being based on the square-law SPICE LEVEL 3 model. The parasitic and noise effects have been neglected in the performed analysis, but it could be estimated that their impact to the overall accuracy of the proposed function synthesizer circuit are relatively small comparing with other causes of errors (approximation error or second-order effects). The overall accuracy of the proposed fourth-order function synthesizer circuit is $0.3 \%$ for an extended range of the input signal.

SPICE simulation $I_{O U T}\left(I_{2}\right)$ for the Multiplier/Divider circuit proposed in Figure 3 is presented in Figure 5. The $I_{O}$ and $I_{1}$ currents have the following values: $I_{O}=$ $50 \mu \mathrm{A}$ and $I_{1}=20 \mu \mathrm{A}$, while the range of $I_{2}$ current was chosen to be between $30 \mu A$ and $100 \mu A$.

A comparison between the simulated and the theoretical estimated results for the proposed current squarer is shown in Figure 6.

SPICE simulation $I_{O U T}\left(I_{1}\right)$ for the Multiplier/Divider circuit proposed in Figure 3 is presented in Figure 7. The $I_{O}$ and $I_{2}$ currents have the following values: $I_{O}=$ $10 \mu \mathrm{A}$ and $I_{2}=100 \mu \mathrm{A}$, while the range of $I_{1}$ current was chosen to be between 0 and $100 \mu A$.

SPICE simulation $I_{O U T}\left(I_{2}\right)$ for the Multiplier/Divider circuit proposed in Figure 3 is presented in Figure 8. The $I_{O}$ and $I_{1}$ currents have the following values: $I_{O}=$

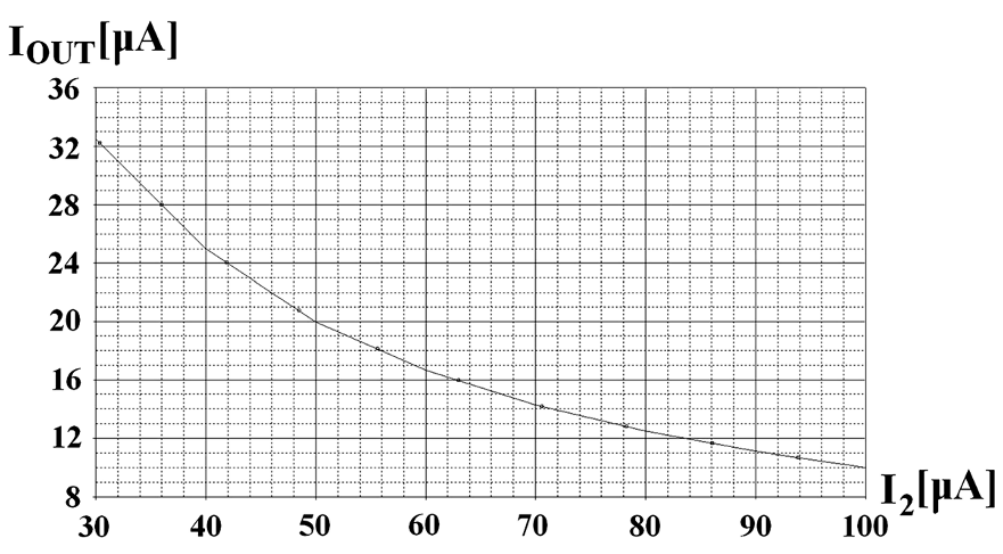

Figure 8 SPICE simulation $I_{\text {OUT }}\left(I_{2}\right)$ for the Multiplier/Divider circuit. 


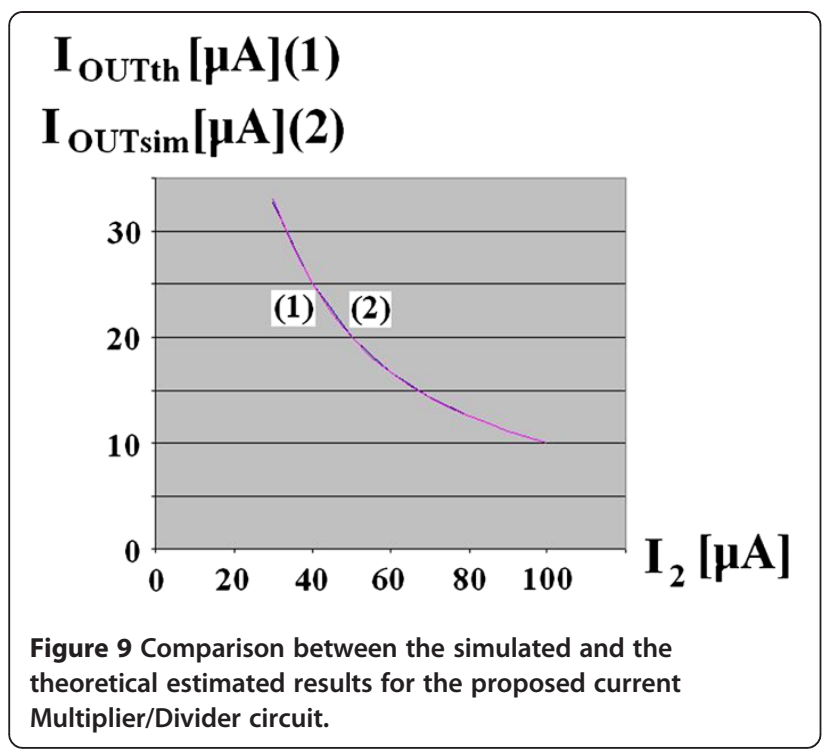

$50 \mu \mathrm{A}$ and $I_{1}=20 \mu \mathrm{A}$, while the range of $I_{2}$ current was chosen to be between $30 \mu A$ and $100 \mu A$.

A comparison between the simulated and the theoretical estimated results for the proposed current Multiplier/Divider circuit is shown in Figure 9. The values of $I_{O}$ and $I_{1}$ currents are $I_{O}=50 \mu \mathrm{A}$ and $I_{1}=20 \mu \mathrm{A}$, while $I_{2}$ current has values between $30 \mu A$ and $100 \mu A$.

As a result of the particular architecture proposed for the realization of the function synthesizer structure, it presents a low-voltage operation (a minimal supply voltage of $I V$ for an implementation in the mentioned technology). This value of the minimal supply voltage was obtained using the theoretical analysis of the proposed function synthesizer circuit $\left(V_{D D \min }=2 V_{S G}+V_{D S s a t}\right)$ and also by performing simulations based on the square-law SPICE LEVEL 3 model.

\section{Methods}

The necessity of implementing a multitude of continuous mathematical functions in CMOS technology has been solved by the original proposed function synthesizer circuit. A very important advantage of this computational structure is mainly related to its large capability of generating continuous mathematical functions: exponential, multiplying/ dividing, or squaring/square-rooting functions. The original operating method was based on a new fourth-order approximation function, having a superior-order polynomial series that match the polynomial series of the approximated function. The current-mode operation and the independence of the circuit performances on the technological errors are responsible for an additional improvement of structure accuracy. The proposed function synthesizer circuit allows a relatively simple implementation in CMOS technology using only two current-mode Multiplier/Divider circuits.
The designed function synthesizer circuit has the advantages of increased modularity and controllability and of minimal design costs per implemented mathematical function. The function synthesizer is designed for implementing in $0.18 \mu \mathrm{m}$ CMOS technology and it is supplied at $I V$. SPICE simulations confirm the theoretical estimated results, showing an accuracy of $0.3 \%$ for an extended range of the input signal. This accuracy is exclusively referring to the precision of generating the previous functions, not to the additional circuits that deserve the proposed function synthesizer structure.

The speed of the original proposed function generator circuit is correlated with its overall accuracy, expecting the necessity of making a tradeoff between the previous performance parameters. Additionally, especially for extreme small values of the approximation error, the noise of the circuit can slightly degrade the circuit performances.

As an immediate application of the function synthesizer circuit, it was proposed a new exponential function generator based on a particular fourth-order approximation function. The circuit has a dynamic range of approximately $33 \mathrm{~dB}$, in the conditions of limiting the error to $1 \mathrm{~dB}$.

\section{Abbreviations}

CMOS: complementary metal oxide semiconductor; MOS: metal oxide semiconductor; VLSI: very large-scale integration.

\section{Competing interests}

The author declares that they have no competing interests.

\section{Acknowledgments}

The author thanks Professor Maurits Ortmanns from Institute of Microelectronics, University of Ulm for reviewing the article and for helpful discussions, the collaboration with him bringing many improvements to the article.

\section{Author's information}

Cosmin Radu Popa was born in Constanta, Romania. He received the B. Eng. degree in electronic engineering and telecommunications in 1997, the M.Sc. degree in microelectronics in 1998, and the Ph.D. degree in electronic engineering and telecommunications with Summa Cum Laude distinction in 2004 from the University Politehnica of Bucharest,

Romania. Currently, he is a lecturer at Faculty of Electronics,

Telecommunications and Information Technology, Bucharest, Romania. In 2006, he earned "In Hoc Signo Vinces" award, Magna Cum Laude

distinction from The National Council of Scientific Research and, in 2008, "In Tempore Opportuno" award from University Politehnica of Bucharest, both from excellence in research activity. From 2010, he is involved in a Postdoctoral Research Fellow with the same faculty. His main area of research is analog and mixed signal integrated circuits for low-power low-voltage applications and analog signal processing. Dr. Popa is author of five books (two of them published with Springer) covering the domains of analog signal processing circuits and, also, of more than 150 papers published in the most important international journals and conference proceedings.

Received: 20 February 2012 Accepted: 23 June 2012

Published: 13 July 2012

\section{References}

1. G Rittenhouse, S Goyal, DT Neilson, S Samuel, Sustainable telecommunications (Technical Symposium at ITU Telecom World, Geneva, Switzerland, 2011), pp. 19-23 
2. F Altiparmak, B Dengiz, AAE Smith, General neural network model for estimating telecommunications network reliability. IEEE Trans. Reliab. 58(1), 2-9 (2009)

3. C Popa, Superior-Order Curvature-Correction Techniques for Voltage References (Springer, New York, 2009)

4. C Popa, Synthesis of Computational Structures for Analog Signal Processing (Springer, New York, 2011)

5. CC Du Preez, S Sinha, M du Plessis, CMOS ECG, EEG and EMG waveform bio-simulator. International Semiconductor Conference 1, 27-29 (2006). September 2006, Sinaia, Romania, pp. 29-38

6. F Pini, K McCarthy, Capacitive instrumentation amplifier for low-power bio potential signal detection (Paper presented at Signals and Systems Conference, Cork, Ireland, 2010), pp. 54-58. 23-24 June 2010

7. C Azzolini, A Ricciardi, A Boi, Very low-cost CMOS audio amplifier for 1-V portable applications (Paper presented at 3rd International Conference on Design and Technology of Integrated Systems in Nanoscale Era, Tozeur, Tunisia, 2008), pp. 25-27. pp. 1-4

8. C Azzolini, A Boni, A 1-V CMOS audio amplifier for low cost hearing aids, in Paper presented at 15th IEEE International Conference on Electronics, Circuits and Systems, 31 August - 3, St (Julian's, Malta, September 2008), pp. 562-565

9. DC Wei, DQ Sun, AA Abidi, A 300-MHz fixed-delay tree search-DFE analog CMOS disk-drive read channel. IEEE J. Solid State Circuits 36(11), 1795-1807 (2001)

10. T Ndjountche, L Fa-Long, C Bobda, A CMOS front-end architecture for harddisk drive read-channel equalizer (Paper presented at IEEE International Symposium on Circuits and Systems, Kobe, Japan, 2005), pp. 2184-2187. vol. 3, 23-26 May

11. C Hwang-Cherng, W Jia-Yu, High CMRR instrumentation amplifier for biomedical applications (Paper presented at 9th International Symposium on Signal Processing and Its Applications, 12-15 February 2007, Sharjah, United Arab Emirates, 2007), pp. 1-4

12. MR Haider, SK Islam, S Mostafa, Z Mo, O Taeho, Low-power low-voltage current readout circuit for inductively powered implant system. IEEE Trans. Biomed. Circuits Syst. 4(4), 205-213 (2010)

13. R Dlugosz, T Talaska, W Pedrycz, R Wojtyna, Realization of the conscience mechanism in CMOS implementation of winner-takes-all self-organizing neural networks. IEEE Trans. Neural Netw. 21(6), 961-971 (2010)

14. IE Ebong, P Mazumder, CMOS and memristor-based neural network design for position detection. Proc. IEEE 99, 1-11 (2011)

15. K Ming-Dou, ESD-aware circuit design in CMOS integrated circuits to meet system-level ESD specification in microelectronic systems (Paper presented at International Conference of Electron Devices and Solid-State Circuits, Tianjin, China, 2011), pp. 1-2. 17-18 November 2011

16. L Seung-Chul, J Young-Deuk, K Jong-Kee, K Jongdae, A 10-bit 205-MS/s 1.0$\mathrm{mm}^{2}$ 90-nm CMOS pipeline ADC for flat panel display applications. IEEE J. Solid State Circuits 42(12), 2688-2695 (2007)

17. N Sudha, K Sridharan, A high-speed VLSI design and ASIC implementation for constructing Euclidean distance-based discrete Voronoi diagram. IEEE Trans. Robot. Autom. 20(2), 352-358 (2004)

18. T Villmann, FM Schleif, B Hammer, Fuzzy Labeled Soft Nearest Neighbor Classification with Relevance Learning (Paper presented at Fourth International Conference on Machine Learning and Applications, Los Angeles, USA, 2005), pp. 11-15. 15-17 December 2005

19. I Triguero, J Derrac, S Garcia, F Herrera, A taxonomy and experimental study on prototype generation for nearest neighbor classification. IEEE Trans. Syst. Man Cybern. C: Appl. Rev. 42(1), 86-100 (2012)

20. A Rodríguez-Vázquez, R Domínguez-Castro, F Jiménez-Garrido, S Morillas, A García-Ortiz, C Utrera, MD Pardo, J Listan, R Romay, in Cellular Nanoscale Sensory Wave Computing - A CMOS Vision System On-Chip with Multi-Core, Cellular Sensory-Processing Front-End, ed. by C. Baatar, W. Porod, T. Roska (Springer, New York, 2010), pp. 129-146

21. C Sawigun, J Mahattanakul, A 1.5 V, wide-input range, high-bandwidth, CMOS four-quadrant analog multiplier (IEEE International Symposium on Circuits and Systems, Washington, USA, 2008), pp. 2318-2321. 18-21 May 2008

22. BC Akshatha, VK Akshintala, Low voltage, low power, high linearity, high speed CMOS voltage mode analog multiplier (Paper presented at 2nd International Conference on Emerging Trends in Engineering and Technology, Nagpur, India, 2009), pp. 149-154. 16-18 December 2009

23. SA Mahmoud, Low voltage low power wide range fully differential CMOS fourquadrant analog multiplier (Paper presented at 52nd IEEE International
Midwest Symposium on Circuits and Systems, Cancun, Mexico, 2009), pp. 130-133. 2-5 August 2009

24. A Naderi, A Khoei, K Hadidi, High speed, low power four-quadrant CMOS current-mode multiplier (Paper presented at 14th IEEE International Conference on Electronics, Circuits and Systems, Marrakech, Morocco, 2007), p. 1311. 11-14 December 2007

25. M Gravati, M Valle, G Ferri, N Guerrini, N Reyes, A novel current-mode very low power analog CMOS four quadrant multiplier (Paper presented at 31st European Solid-State Circuits Conference, Grenoble, France, 2005), pp. 495498. 12-16 September 2005

26. C Sawigun, WA Serdijn, Ultra-low-power, class-AB. CMOS four-quadrant current multiplier. Electron. Lett. 45(10), 483-484 (2009)

27. R Hidayat, K Dejhan, P Moungnoul, Y Miyanaga, OTA-based high frequency CMOS multiplier and squaring circuit (Paper presented at International Symposium on Intelligent Signal Processing and Communications Systems, Bangkok, Thailand, 2009), pp. 1-4. 8-10 Ferbruary 2009

28. W Machowski, S Kuta, J Jasielski, W Kolodziejski, Quarter-square analog fourquadrant multiplier based on CMOS invertes and using low voltage high speed control circuits (Paper presented at 17th International Conference Mixed Design of Integrated Circuits and Systems, Wroclaw, Poland, 2010), pp. 333336. 24-26 June 2010

29. M Ehsanpour, P Moallem, A Vafaei, Design of a novel reversible multiplier circuit using modified full adde (Paper presented at International Conference on Computer Design and Applications, Qinhuangdao, China, 2010), pp. V3230-V3-234. vol. 3, 25-27 June 2010

30. A Naderi, H Mojarrad, H Ghasemzadeh, A Khoei, K Hadidi, Four-quadrant CMOS analog multiplier based on new current squarer circuit with high-speed (IEEE International Conference on "Computer As A Tool, Saint Petersburg, Russia, 2009), pp. 282-287. 18-23 May 2009

31. C.H. Kao, C.C. Tseng, C.S. Hsieh, Low-voltage exponential function converter. IEE Proc. Circuits Dev. Syst. 152(5), 485-487 (2005)

32. S Ethier, M Sawan, Exponential Current pulse generation for efficient very high-impedance multisite stimulation. IEEE Trans. Biomed. Circuits Syst 99, 1-9 (2010)

33. H Hedayati, B Bakkaloglu, A $3 \mathrm{GHz}$ wideband $\sum \Delta$ fractional-N synthesizer with voltage-mode exponential CP-PFD (Paper presented at IEEE Radio Frequency Integrated Circuits Symposium, Boston, USA, 2009), pp. 325-328. 7-9 June 2009

34. D Moro-Frias, MT Sanz-Pascual, CA de la Cruz-Bias, Linear-in-dB Variable Gain Amplifier with PWL exponential gain control (Paper presented at IEEE International Symposium on Circuits and Systems, Paris, France, 2010), pp. 2824-2827. 2010

35. CA De La Cruz Blas, O Feely, Limit cycle behavior in a class-AB second-order square root domain filter, in Paper presented at 15th IEEE Internationa Conference on Electronics, Circuits and Systems, 31 August - 3, St (Julian's, Malta, September 2008), pp. 117-120

36. B Boonchu, W Surakampontom, A CMOS current-mode squarer/rectifier circuit (Paper presented at International Symposium on Circuits and Systems, Bangkok, Thailand, 2003), pp. |-405-|-408. vol. 1, 25-28 May 2003

37. CA De La Blas, A Lopez, A novel two quadrant MOS translinear Squarerdivider cell, in Paper presented at 15th IEEE International Conference on Electronics, Circuits and Systems, 31 August - 3 Sptember, St (Julian's, Malta, 2008), pp. 5-8

38. CA De La Cruz-Blas, AJ Lopez-Martin, A Carlosena, 1.5-V square-root domain second-order filter with on-chip tuning. IEEE Trans. Circuits Syst. I: Regular Papers 52(10), 1996-2006 (2005)

39. G Raikos, S Vlassis, Low-voltage CMOS voltage squarer (Paper presented at 16th IEEE International Conference on Electronics, Circuits, and Systems, Yasmine Hammamet, Tunisia, 2009), pp. 159-162. 13-16 December 2009

40. V Garofalo, M Coppola, D De Caro, E Napoli, N Petra, AGM Strollo, A novel truncated squarer with linear compensation function (Paper presented at IEEE International Symposium on Circuits and Systems, Paris, France, 2010), pp. 4157-4160. 30 May - 2 June 2010

41. A Kircay, MS Keserlioglu, Novel current-mode second-order square-rootdomain highpass and allpass filter (Paper presented at International Conference on Electrical and Electronics Engineering, Bursa, Turkey, 2009), pp. 242-246. 5-8 November 2009

42. C Popa, A New FGMOST Euclidean Distance Computational Circuits Based on Algebraic Mean of the Input Potentials (Springer, Berlin, 2009), pp. 459-466. Lecture Notes in Computer Science 
43. A Hyo-Jin, C Chang-Seok, L Hanho, High-speed low-complexity Folded Degree-Computationless Modified Euclidean algorithm architecture for RS decoders (Paper presented at 12th International Symposium on Integrated Circuits, Singapore, Singapore, 2009), pp. 582-585. 14-16 December 2009

44. MT Abuelma'atti, NM Al-Yahia, An improved universal CMOS current-mode analog function synthesizer (Paper presented at International Symposium on Integrated Circuits, Singapore, 2007), pp. 580-583. 26-30 September 2007

45. MT Abuelma'atti, Universal CMOS current-mode analog function synthesizer. IEEE Trans. Circuits Syst. I: Fund. Theory Appl 49(10), 1468-1474 (2002)

46. MT Abuelma'atti, A translinear current-mode programmable analog exponential function synthesizer (Paper presented at Eleventh International Conference on Microelectronics, Kuwait, 1999), pp. 209-212. 22-24 November 1999

47. C Psychalinos, S Vlassis, A systematic design procedure for square-root domain circuits based on the signal flow graph approach. IEEE Trans. Circuits Syst. I: Fund. Theory Appl. 49(12), 1702-1712 (2002)

48. GA Baker, P Graves-Morris, Padé Approximants (Cambridge University Press (Cambridge, MA, 1996)

doi:10.1186/1687-6180-2012-146

Cite this article as: Popa: High-accuracy function synthesizer circuit with applications in signal processing. EURASIP Journal on Advances in Signal Processing 2012 2012:146

\section{Submit your manuscript to a SpringerOpen ${ }^{\circ}$ journal and benefit from:}

- Convenient online submission

- Rigorous peer review

- Immediate publication on acceptance

- Open access: articles freely available online

- High visibility within the field

- Retaining the copyright to your article 